\title{
Editorial
}

Oncology

\section{Continuation or Reintroduction of an Antineoplastic Strategy after Documented Disease Progression}

\author{
Maurie Markman \\ Cancer Treatment Centers of America, Eastern Regional Medical Center, Philadelphia, Pa., USA
}

It is increasingly acknowledged that our understanding of the complexity of cancer biology is limited. This recognition has resulted in the recent rather rapid development of often paradigm-changing concepts of how cancer develops and progresses and how it can be optimally managed. The profoundly oversimplified view of a given cancer as a biologically homogeneous entity within a class of illnesses (e.g., breast, lung, and others) or within an individual patient has essentially been abandoned with the realization that malignant disease, even within a single patient, is remarkably heterogeneous in its molecular makeup.

As an extension of this realization, it is relevant to question, if not outright discard, the long-standing concept within the clinical oncology arena that 'progression' in a single mass lesion in a patient with a malignancy should be viewed as an indication that cancer throughout the body is now 'resistant' to a given antineoplastic strategy. While such a determination is rational when attempting to define an objectively finite endpoint within a given clinical trial (e.g., time to the initial episode of documented disease progression for patients on regimen A compared to those on regimen $\mathrm{B}$ ), the clinical utility of this endpoint outside the confines of a study is increasingly questionable.

\section{KARGER}

(c) 2013 S. Karger AG, Basel

0030-2414/13/0856-0348\$38.00/0

E-Mail karger@karger.com

www.karger.com/ocl
It has long been recognized that patients in a number of clinical settings (e.g., lymphomas, ovarian cancer) who respond to a particular cytotoxic antineoplastic agent/ regimen and who recur at some variously defined time interval after treatment discontinuation may experience a second (or third) response to the same or similar drug(s) [1]. In fact, such an approach would be considered standard of care with a relatively prolonged treatment-free interval between the time of treatment discontinuation and the development of documented disease recurrence.

Recent data provide support for the continuation of several more 'targeted' antineoplastic agents after documented disease progression where the drug had been employed as a component of the prior treatment strategy [2, 3]. However, to date, there remains limited published experience utilizing this basic approach. Therefore, the report by Naing et al. [4] is an important addition to this literature as it strongly suggests the potential substantial therapeutic utility of employing this strategy to disease management in carefully considered clinical settings.

Similar to the experience with cytotoxic drugs, these investigators considered the reintroduction of a targeted antineoplastic in patients whose cancers had previously exhibited evidence of biological activity and had a rather prolonged period of time between the initial use of the 
strategy and consideration of retreatment (median 92 weeks). While the sample size in this report was limited $(\mathrm{n}=11)$, the investigators have provided important data that may be highly relevant for oncologists considering options for individual patients in similar clinical circumstances.

As cancer is increasingly viewed in many settings as a serious chronic disease, it may be quite appropriate to consider retreatment with a strategy that was previously demonstrated to produce a favorable clinical impact at an earlier point in the patient's cancer journey. As basic and translational laboratory research has unequivocally revealed the heterogeneity in the fundamental biology of individual cancers and the molecular changes that occur over relatively short periods of time and under the 'stress' of active treatment, it should be clear that our old view of an unalterable 'fixed' pattern of clinical resistance (e.g., progression while on or following discontinuation of drug A means the cancer present within the patient is permanently resistant of $\operatorname{drug} A$ ) must be seriously challenged and modified.

\section{References}

Continuation or Reintroduction of an Antineoplastic Strategy
Hennessy BT, Coleman RL, Markman M: Ovarian cancer. Lancet 2009;374:13711382.

-2 Kubicka S, Greil R, Andre T, et al: Bevacizumab plus chemotherapy continued beyond first progression in patients with metastatic colorectal cancer previously treated with bevacizumab plus chemotherapy: ML18147 study KRAS subgroup findings. Ann Oncol 2013;24:2342-2349.
Von Minckwitz G, du Bois A, Schmidt M, et al: Trastuzumab beyond progression in human epidermal growth factor receptor 2-positive advanced breast cancer: a German Breast Group 26/Breast International Group 03-05 Study. J Clin Oncol 2009;27:1999-2006.

4 Naing A, Agarwal R, Falchook G, Hong DS, Janku F, Wheler J, Fu S, Kurzrock R: Retreatment after secondary resistance or mixed response: a pilot study. Oncology 2013;85:350355. 